$R_{i}$

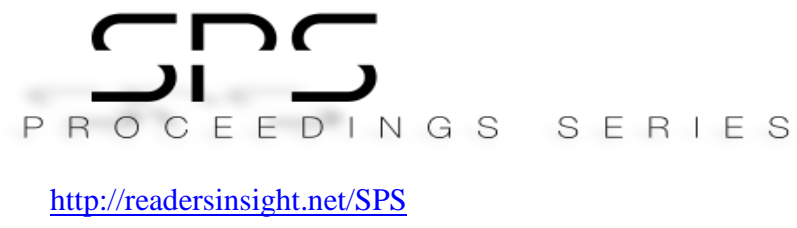

\title{
EFFICIENT ANALYTICAL APPROACH FOR NONLINEAR SYSTEM OF ADVANCED LORENZ MODEL
}

\author{
Aminu Barde \\ Department of Mathematical Sciences \\ Abubakar Tafawa Balewa University Bauchi \\ Nigeria \\ Department of Mathematical Sciences \\ Universiti Teknology Malaysia 81310 Skudai, Johor \\ Malaysia \\ Normah Maan* \\ Department of Mathematical Sciences \\ Universiti Teknologi Malaysia 81310 Skudai, Johor \\ Malaysia
}

*Corrosponding author's Email: bardealamin@yahoo.com

Peer-review under responsibility of 4th Asia International Multidisciplinary Conference 2020 Scientific Committee http://connectingasia.org/scientific-committee/ (C) 2020 Published by Readers Insight Publisher, lat 306 Savoy Residencia, Block 3 F11/1,44000 Islamabad. Pakistan,

editor@readersinsight.net

This is an open access article under the CC BY-NC-ND license (http://creativecommons.org/licenses/by-nc-nd/4.0/). 


\section{$R_{i}$}

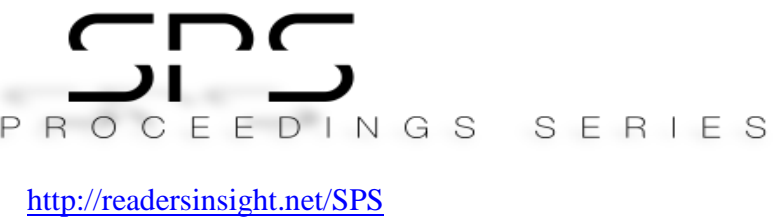

\section{Rese a r ch H igh I ight s}

This work proposed a new analytical approach for solving a famous model from mathematical physics, namely, advanced Lorenz system. The method combines the Natural transform and Homotopy analysis method, and it's have been suggested for the solution of various kinds for the systems of nonlinear delay differntial equations (DDEs). This technique generates solution in a polynomial series, where the modification of He's polynomial is successfully derived for the computions of nonlinear functions of the Lorenz system. By choosing an optimal value of auxiliary parameters the more precise approximation of the model is achieved at a maximum of three iterational number of terms. Some figures are used to demonstrate the accuracy of the result based on the residual error function. Therefore, the approach gives rise to an easy and straightforward means of solving these models analytically. Hence, it can be used in finding solutions to other forms of nonlinear problems.

Keywords:- Natural Transform, HomotopyAnalysis Method, He's Polynomial, Advanced, Lorenz System.

\section{Graphical A bstract}

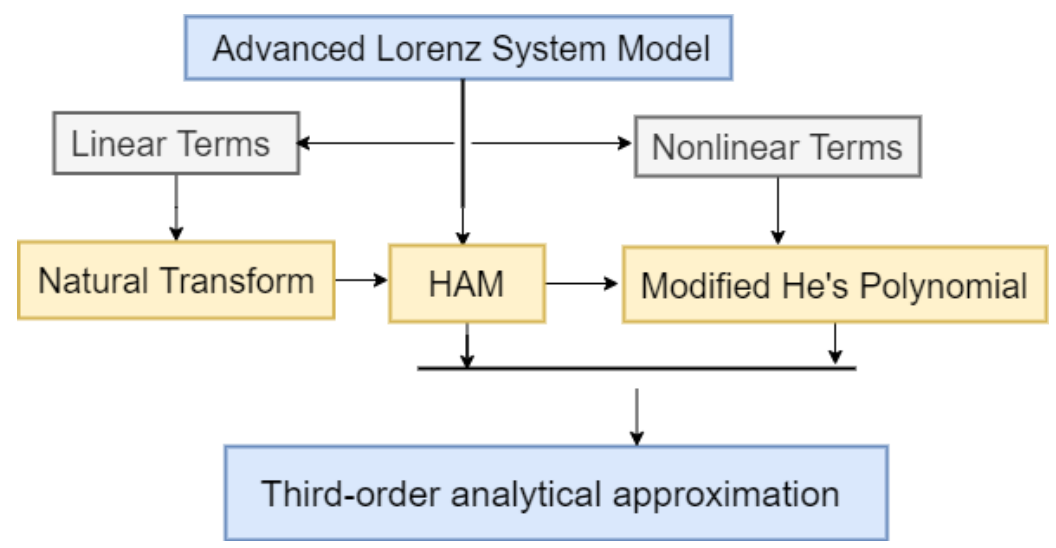

\section{Research Objectives}

Delay differential equations rise in numerous fields in applied sciences and is very significant in the mathematical modelling of problems from real-life phenomena. Several problems from various fields of studies contain a delay element. The few of such include the biological species living together [1], the dynamics model of prey-predator which gives rise to delayed Volterra integro-differential equations [2] and the problems in population dynamics lead to the formation of delay logistic equation [3]. Partly due to the nature of the infinite-dimensional state they possess, methods of solving ODEs are not generally applied to DDEs. Therefore, the 
$R_{i}$

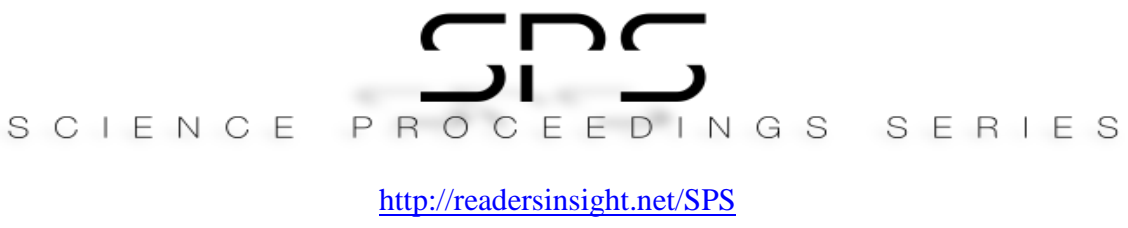

http://readersinsight.net/SPS

analytical solutions of these models are hardly ever available. Hence, they are mostly solved by numerical methods. In this research, an efficient analytical approach is applied to solve a model from mathematical physics namely the Advanced Lorenz system. This approach is developed in [4] for an efficient analytical approximation of different forms of the systems of nonlinear retarded delay differential equations (RDDEs). Therefore, the aim here is to find a better approximation analytically of this model using the proposed technique.

\section{Methodology}

The present work focus to obtain a better analytical approximation for Advanced Lorenz system model by using the approach developed in [4] for the systems of nonlinear RDDEs. This technique is from the combinational form of Natural transform (NT) and Homotopy analysis method (HAM) where the modification of He's polynomial is successfully derived for the computions of nonlinear functions. This technique provides solutions to various forms of nonlinear systems of RDDEs in a polynomial series that converged rapidly to an exact or approximate solutions using a maximum of three iteratioal numbers of terms. The idea here is use the Natural transform as a linear operator which is applied to obtain the simplified form of the linear term of such model where the concept HAM is used to construct the generating function for the computation of iterational terms. Lastly, the modified He's polynomial is defined for the calculating the nonlinear functions in the generating function constructed.

\section{Results}

Advanced Lorenz model is introduced by Xiao-hong et al. [5] and it is an advancement of the model proposed by Lorenz in [6] for atmospheric convection. So, according to Ansari and Dasi [7] the system of this model can be obtained as follows.

$$
\begin{aligned}
& x_{1}^{\prime}(t)=20\left[x_{2}(t)-x_{1}(t)\right]+3 x_{1}(t)(t-\tau) \\
& x_{2}^{\prime}(t)=14 x_{1}(t)+\frac{53}{5} x_{2}(t)-x_{1}(t) x_{3}(t) \\
& x_{3}^{\prime}(t)=x_{1}^{2}(t)-\frac{14}{5} x_{3}(t),
\end{aligned}
$$

with initial condition

$$
x_{1}(0)=-20, \quad x_{2}(0)=8, \quad x_{3}(0)=20
$$

To find the approximation for the system in Eq. (1) based on the proposed technique, The NT of Equation should be first taking, and then by making the further simplification using the given initial condition the simplified form of the model is obtained as 


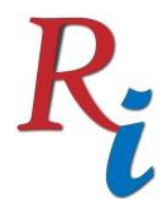

\section{T)}

SCIENCE PROCEEDINGS SERIES

http://readersinsight.net/SPS

$$
\begin{aligned}
& \mathbb{N}^{+}\left[x_{1}(t)\right]+\frac{20}{s}+\frac{20}{s}\left[x_{1}(t)-x_{2}(t)\right]-\frac{3 u}{3} \mathbb{N}^{+}\left[x_{1}(t-\tau)\right]=0 \\
& \mathbb{N}^{+}\left[x_{2}(t)\right]-\frac{8}{s}-\frac{14 u}{s} \mathbb{N}^{+}\left[x_{1}(t)\right]-\frac{53}{5 s} \mathbb{N}^{+}\left[x_{2}(t)\right]+\frac{u}{s} \mathbb{N}^{+}\left[x_{1}(t) x_{2}(t)\right]=0 \\
& \mathbb{N}^{+}\left[x_{3}(t)\right]-\frac{20}{s}+\frac{14 u}{5 s} \mathbb{N}^{+}\left[x_{3}(t)\right]-\frac{u}{s} \mathbb{N}^{+}\left[x_{1}^{2}(t)\right]=0
\end{aligned}
$$

So, by following the procedure in [4] the recursive relation of the model can be obtained as

$$
\begin{gathered}
x_{1, m}(t)=\left(\chi_{m}+h_{1}\right) x_{1, m-1}(t)+h_{1}\left(1-\chi_{m}\right) \mathbb{N}^{-}\left[\frac{20}{s}\right]+h_{1} \mathbb{N}^{-}\left\{\frac{u}{s} \mathbb{N}^{+}\left[R_{1}\left(x_{1, m-1}, x_{2, m-1}\right)\right]\right\} \\
x_{2, m}(t)=\left(\chi_{m}+h_{2}\right) x_{2, m-1}(t)-h_{2}\left(1-\chi_{m}\right) \mathbb{N}^{-}\left[\frac{8}{s}\right]-h_{2} \mathbb{N}^{-}\left\{\frac { u } { s } \mathbb { N } ^ { + } \left[R_{2}\left(x_{1, m-1}, x_{2, m-1}\right)+\right.\right. \\
\left.\left.H_{2, m-1}\left(x_{1,1} x_{3,1}, \ldots x_{1, N} x_{3, N}\right)\right]\right\} \\
x_{3, m}(t)=\left(\chi_{m}+h_{3}\right) x_{3, m-1}(t)-h_{3}\left(1-\chi_{m}\right) \mathbb{N}^{-}\left[\frac{20}{s}\right]+h_{3} \mathbb{N}^{-}\left\{\frac { u } { s } \mathbb { N } ^ { + } \left[R_{3}\left(x_{3, m-1}(t)\right)+\right.\right. \\
\left.\left.H_{3, m-1}\left(x_{1,1}(t), x_{1,2}(t), \ldots x_{1, N}(t)\right)\right]\right\} \quad m \geq 1,
\end{gathered}
$$

where the nonlinear functions $x_{1}(t) x_{2}(t)$ and $x_{1}^{2}(t)$ are respectively calculated as the series of modified He's polynomials defined as

$$
H_{2, m}\left(x_{1,1} x_{3,1}, \ldots x_{1, N} x_{3, N}\right)=\frac{1}{m !} \frac{\partial^{m}}{\partial q^{m}} F_{2}\left(\sum_{p=0}^{m} q^{p}\left(x_{1, p}(t), x_{2, p}(t)\right)\right)
$$

and

$$
H_{3, m}\left(x_{1,1}(t), x_{1,2}(t), \ldots x_{1, N}(t)\right)=\frac{1}{m !} \frac{\partial^{m}}{\partial q^{m}} F_{3}\left(\sum_{p=0}^{m} x_{1, p}(t) q^{p}\right) .
$$

\section{Findings}

From the given intial condition the intial approximation can be chosen as

$$
x_{1,0}(t)=-20, \quad x_{2,0}(t)=8 \text { and } x_{3,0}(t)=20
$$

So, from Eq. (3) at $h_{1}=-0.997$ and $h_{2}=h_{3}=-1$, the third-order approximate solution of this model is given as follows:-

$$
x_{1}(t) \approx \sum_{m=0}^{3} x_{1, m}=32647.5 t^{3}-2182.643 t^{2}+498.4977 t-20
$$




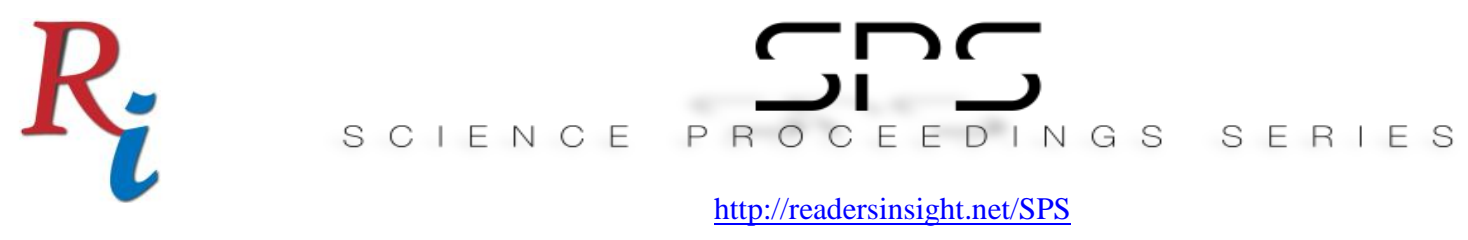

$$
\begin{aligned}
& x_{2}(t) \approx \sum_{m=0}^{3} x_{2, m}=-112116.777 t^{3}+3029.94 t^{2}+204.8 t+8 \\
& x_{3}(t) \approx \sum_{m=0}^{3} x_{3, m}=122476.17 t^{3}-1045.6 t^{2}+498.4977 t+20
\end{aligned}
$$

Therefore, based on the result obtained the most important thing about the proposed method is to make a good choose of the initial approximation, and this will accelerate the convergent for the series solutions and also determine the best set of function to be used, and to have better approximation from few numbers of iterations.

\section{Acknowledgement}

We thanks the Transdisciplinary Research Grant Scheme (TRGS) for their financial support provided under the resarch activities of Characterization of Spatio-temporal Marine Microalgae Ecological Impact Using Multi-sensor Over Malaysia Waters particularly to sub-work of Mathematical Modeling of Harmful Algal Blooms (HABs) in Malaysia Waters (R.J130000.7809.4L854) and also the Fundamental Research Grant Scheme (FRGS) with title Tumor-Hyperthermia Immunotherapy Model with Optimal Temperature Control Strategy (5F220) which is funded by the Ministry of Education, Malaysia. Our grateful to Universiti Teknologi Malaysia for giving the required assistance and services for the succesfull conduct of the work.

\section{REFERENCES}

1. Shakeri, F. and M. Dehghan, Solution of a model describing biological species living together using the variational iteration method. Mathematical and Computer Modelling, 2008. 48(5-6): p. 685-699.

2. Shakourifar, M. and M. Dehghan, On the numerical solution of nonlinear systems of Volterra integro-differential equations with delay arguments. Computing, 2008. 82(4): p. 241.

3. Dehghan, M. and R. Salehi, Solution of a nonlinear time-delay model in biology via semianalytical approaches. Computer Physics Communications, 2010. 181(7): p. 1255-1265.

4. Barde, A. and N. Maan, Efficient Analytical Approach for Nonlinear System of Delay Differential Equations. Computer Science, 2019. 14(3): p. 693-712. 

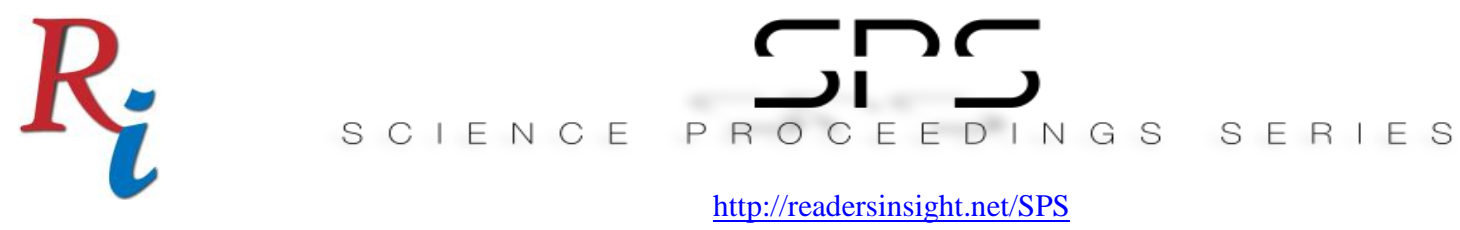

5. Xiao-hong, Z., C. Zhi-yong, and Z. Yuan-yuan. Synchronization and circuit experiment simulation of chaotic time-delay systems. in 2009 Pacific-Asia Conference on Circuits, Communications and Systems. 2009. IEEE.

6. Lorenz, E.N., Deterministic nonperiodic flow. Journal of the atmospheric sciences, 1963. 20(2): p. 130-141.

7. Ansari, S.P. and S. Das, Projective synchronization of time-delayed chaotic systems with unknown parameters using adaptive control method. Mathematical methods in the applied sciences, 2015. 38(4): p. 726-737.

Author's Biography

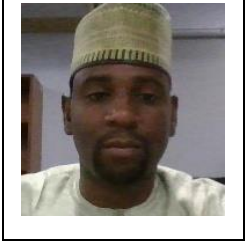

Aminu Barde works with Abubakar Tafawa Balewa University, Bauchi as a lecturer from 2009 to date. He received his BSc Mathematics from Bayero University, Kano in 2006 and obtained his MSc in 2014 from Abubakar Tafawa Balewa University, Bauchi. He is currently a PhD student at Universiti Teknologi Malaysia. Aminu Barde has conducted various research in applied mathematics and the current research area is delay differential equations.

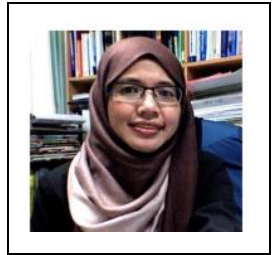

Normah Maan is an Associate Professor of Department of Mathematical Sciences at Universiti Teknologi Malaysia. She received her BSc (Hons) Mathematics from the University of Sheffield, United Kingdom, in 1991, which then continued her Ph.D. study at University Teknologi Malaysia. She leads the Dynamical System Modeling Research Group at her faculty and has conducted various research in Dynamical Modeling and Analysis. Her current research projects are Mathematical Modelling of Tumor-Immune Interaction, Harmful Algal Bloom, Breast Cancer, and Dengue by Delay Differential Equations and Partial Differential Equations. Besides that, her interest area also on problems, which involved uncertainty that used Fuzzy Approach 